\title{
Salaire au mérite et contrats des médecins hospitaliers
}

Les contrats des médecins hospitaliers accordent une place de plus en plus importante aux bonus liés aux objectifs, au détriment des honoraires de l'activité médicale privée. Lors de la dernière séance d'information de la FMH consacrée à SwissDRG, le Prof. Margit Osterloh a expliqué pourquoi les bonus liés aux objectifs pouvaient être contre-productifs pour les tâches complexes et brider la motivation intrinsèque.

\section{Beatrix Meyer}

Responsable du département Tarifs et économie de la santé pour les médecins hospitaliers

\begin{abstract}
Augmentation de la rémunération au mérite Ne datant pas d'hier chez les médecins hospitaliers, la part de salaire variable s'élève aujourd'hui en Suisse à 30\% dans les contrats de médecin-chef [1] Toutefois, cette part variable de la rémunération se décline en plusieurs composantes comme les honoraires de l'activité médicale privée et des expertises, les participations à des pools, ou depuis quelques temps aussi, les gratifications liées aux objectifs. Une étude menée en Allemagne montre que la part de la rémunération pour les prestations médicales privées a perdu de son importance au cours de ces dernières années alors que les bonus liés aux objectifs augmentent. Chez les médecins-chefs allemands, le taux de contrats nouvellement signés et spécifiant une grati-
\end{abstract}

\section{Le salaire au mérite permet d'augmenter le rendement du travail à la chaîne.}

fication est passé de moins de 5\% en 1995 à quasiment $50 \%$ aujourd'hui. Cette tendance ne concerne pas uniquement les médecins-chefs mais aussi les chefs de clinique, les spécialistes, et même les médecins-assistants dont 5\% déjà ont signé une telle convention [2]. En Suisse aussi, les gratifications liées aux objectifs devraient gagner en importance au détriment d'autres parts variables du salaire telles que les honoraires de l'activité médicale privée.

\section{Liens fréquents entre objectifs et quantité}

Les bonus sont souvent définis en fonction d'objectifs quantifiés prenant en compte par exemple le nombre de cas traités, le case-mix de la clinique ou le succès de la clinique. La presse allemande critique principalement le risque de créer des incitatifs erronés débouchant notamment sur une augmentation du volume par des traitements inutiles. Les objectifs sont aussi en partie liés à des critères de qualité dont l'évaluation n'est cependant pas encore en place dans divers secteurs des hôpitaux. Par ailleurs, le paiement à la performance (ou P4P, pour Pay for Performance) dans le domaine de la santé fait l'objet d'un débat controversé [3].

\section{Les bonus augmentent le rendement des tâches simples}

Dans ce contexte, la FMH a voulu connaitre l'avis d'une économiste renommée à propos des bonus liés aux objectifs pour les médecins hospitaliers; c'est la raison pour laquelle elle a invité le Prof. Margit Osterloh de l'Université de Warwick (UK), et professeure émérite de l'Université de Zurich, à sa séance d'information destinée aux présidents des sociétés de discipline et des organisations faîtières, et à leurs délégués en charge des DRG.

En introduction à son exposé [4], le Prof. Osterloh a rappelé que dans le paiement à la performance, la variabilité du salaire est liée à la réalisation d'objectifs fixés au préalable. Dans le cadre d'activités simples, ce mode de rémunération peut engendrer une amélioration du rendement, pour autant qu'il soit possible de définir des objectifs clairs et que le résultat, c'est-à-dire la réalisation des objectifs, puisse être quantifié (mesurable et imputable) et que le contexte et les conditions qu'ils impliquent puissent être contrôlés. C'est ainsi par exemple que l'introduction du salaire au mérite dans les chaînes de fabrication automobile a permis d'obtenir une amélioration du rendement.

\section{Effets contre-productifs lors de tâches complexes}

En revanche, les activités complexes qui se distinguent par une asymétrie d'informations peuvent subir les effets indésirables du paiement à la performance, comme l'a démontré le Prof. Osterloh avec diverses études empiriques. En effet, la rémunération au mérite incite à se concentrer sur les tâches facilement mesurables donnant droit à une gratification confor- 


\section{Références}

1 Statistique AMDHS 2012.

2 Kienbaum. Vergütungsreport 2012, Führungs- und Fachkräfte in Krankenhäusern, 14. Ausgabe. Kienbaum Management Consultants GmbH, Gummersbach;2012.

3 Veit $\mathrm{C}$ et al. Pay-for-Performance im Gesundheitswesen: Sachstandsbericht zu Evidenz und Realisierung sowie Darlegung der Grundlagen für eine künftige Weiterentwicklung. Gutachten im Auftrag des Bundesministeriums für Gesundheit, BOS-Institut für Qualität und Patientensicherheit;2012.

$4 \quad$ www.fmh.ch $\rightarrow$ Tarifs hospitaliers $\rightarrow$ Publications $\rightarrow$ Séance d'information de la FMH: SwissDRG et salaire au mérite pour les médecins hospitaliers $\rightarrow$ Salaire variable en fonction de la performance: contre-productif pour les médecins hospitaliers.

5 Chen $\mathrm{T}$ et al. The unintended consequence of diabetes mellitus pay-for-performance (P4P) program in Taiwan: are patients with more comorbidities or more severe conditions likely to be excluded from the $\mathrm{P} 4 \mathrm{P}$ program? Health Service Research. 2011;46(1Pt1): 47-60.

6 Gneezy U, Rustichini A Pay enough or don't pay at all. Quarterly Journal of Economics. 2000;115(2): 791-810.

7 Gneezy U, Rustichini A. A fine is a price. Journal of Legal Studies. 2000;29:1-18

8 McGraw K0, McCullers J C. Evidence of detrimental effect of extrinsic incentives on breaking a mental set. Journal of Experimental Social Psychology. 1979; 15:285-94; Ariely D, Gneezy U, Mazar N. Large stakes and big mistakes. Review of Economic Studies. 2009;76:451-69.

9 Eijkenaar F et al. Effects of pay for performance in health care: A systematic review of systematic reviews. Health Policy. 2013;110:115-30.

10 Code de déontologie de la FMH, art. 31, www.fmh.ch $\rightarrow$ De la FMH $\rightarrow$ Bases juridiques $\rightarrow$ Code de déontologie mément aux objectifs fixés, au détriment des tâches délicates ou difficilement quantifiables. En outre, on note une certaine distorsion de la perception à son propre avantage. Le paiement à la performance peut également créer des incitatifs en vue de manipuler le système. C'est ainsi par exemple qu'une étude a montré que les patients âgés et gravement malades ont une plus forte probabilité d'être exclus d'un programme pour diabétiques que des patients jeunes et en meilleure santé [5]. Le Prof. Osterloh a également mentionné d'autres exemples, notamment le cas d'un CEO qui, grâce aux avantages de sa fonction,

\section{Les retours d'information améliorent déjà le résultat.}

avait pu se fixer des objectifs simples. Lorsque la société a dû déposer le bilan, le CEO a quand même touché un bonus de plus de deux millions d'euros car il avait atteint les objectifs fixés. Le paiement à la performance peut également provoquer une autosélection, ou plus exactement un «effet mercenaire», c'est-à-dire qu'il attire les personnes à forte motivation extrinsèque. Plus le nombre de «mercenaires» est élevé dans une entreprise, moins les personnes avec une motivation pro-sociale sont disposées à œuvrer pour le bien de la communauté. Le paiement à la performance peut donc aliéner la motivation intrinsèque des employés.

\section{Preuves empiriques de la perte de motivation intrinsèque}

En s'appuyant sur différentes études, le Prof. Osterloh relève que le paiement à la performance peut réduire la motivation intrinsèque, notamment en raison de la pression externe ressentie comme un facteur de contrôle. A titre d'exemple, elle cite le cas d'élèves qui, sans la moindre gratification, ont récolté nettement plus de fonds pour la lutte contre le cancer que ceux qui ont touché un petit bonus. Si un bonus à hauteur de 1\% des dons recueillis leur était promis, la somme récoltée baissait d'un bon tiers. Une fois que le bonus a été fixé à 10\%, leur engagement a de nouveau nettement augmenté, sans toutefois jamais retrouver le niveau initial [6]. Dans un

Informations complémentaires sur la séance d'information de la FMH

Vous trouverez les documents de séance sur le site www.fmh.ch $\rightarrow$ TARIFS HOSPITALIERS $\rightarrow$ Publications $\rightarrow$ Séance d'information de la FMH: SwissDRG et salaire au mérite pour les médecins hospitaliers. autre exemple, elle mentionne une garderie d'enfants qui a mis en place une pénalité financière pour inciter les parents à venir chercher leurs enfants à l'heure. Mais c'est l'effet inverse qui s'est produit: les parents ont été nettement moins ponctuels qu'au préalable. Lorsque la garderie a de nouveau supprimé la pénalité pour mettre fin à cette expérience, les parents n'ont pas retrouvé leur ponctualité initiale. La motivation intrinsèque avait été irrémédiablement ébranlée [7]. Une autre étude a en outre mis en lumière que le paiement à la performance prolongeait de 50\% le temps requis pour réaliser une tâche demandant de la créativité [8].

\section{Manque d'évidence en faveur du paiement} à la performance dans le domaine de la santé La méta-analyse évoquée par le Prof. Osterloh conclut à un manque de données vérifiées pour recommander l'implantation du salaire au mérite à l'ensemble du domaine de la santé. En revanche, il existe des preuves que les retours d'informations à eux seuls améliorent le résultat et que le paiement à la performance ne provoque aucune amélioration supplémentaire [9]. C'est la raison pour laquelle le Prof. Osterloh conseille de renoncer à toute forme de rémunération incluant des bonus liés aux objectifs, qui peuvent notamment s'avérer contre-productifs. En revanche, un feed-back de soutien par un pair, des gratifications et des distinctions symboliques encouragent une bonne prestation.

\section{Une ligne directrice: le Code de déontologie de la FMH}

Le cadre général existe depuis longtemps, les médecins pouvant en effet se référer au Code de déontologie de la FMH lors de la négociation de contrats. Celui-ci stipule que «Lors de la conclusion d'un contrat, le médecin évite que, dans son activité médicale, un tiers non-médecin puisse lui imposer une action contraire à sa conscience professionnelle. Il refuse notamment tout engagement de fournir certaines prestations médicales ou d'atteindre un certain chiffre d'affaires.» [10] Afin de suivre l'évolution relative aux bonus dans les contrats, la FMH a complété son questionnaire concernant les tâches administratives, la liberté thérapeutique et les effets de SwissDRG. La question des bonus liés aux objectifs sera également traitée lors de la prochaine rencontre du 17 septembre 2013 entre les délégués en charge des DRG auprès des sociétés de discipline et des organisations faîtières. Il s'agira notamment de discuter des éventuelles recommandations détaillées à soumettre aux instances décisionnelles de la FMH. 